\title{
Quality of HIV laboratory testing in Tanzania: a situation analysis
}

\author{
G.S. MFINANGA ${ }^{1}$, B. MUTAYOBA ${ }^{1 *}$, G. MBOGO ${ }^{2}$, A. KAHWA ${ }^{1}$, G. KIMARO ${ }^{2}$, \\ P. P. MHAME ${ }^{2}$, C. MWANGI ${ }^{3}$, M.N. MALECELA ${ }^{2}$ and A.Y. KITUA ${ }^{2}$ \\ ${ }^{1}$ Muhimbili Medical Research Centre, P.O. Box 3436, Dar es Salaam, Tanzania \\ ${ }^{2}$ National Institute for Medical Research, Headquarters, P. O. Box 9653, Dar es Salaam, Tanzania \\ ${ }^{3}$ Centers for Disease Prevention and Control, Dar es Salaam, Tanzania
}

\begin{abstract}
Tanzania is scaling up prevention, treatment, care and support of individuals affected with HIV. There is therefore a need for high quality and reliable HIV infection testing and AIDS staging. The objective of this study was to assess laboratories capacities of services in terms of HIV testing and quality control. A baseline survey was conducted from December 2004 to February 2005 in 12 laboratories which were conveniently selected to represent all the zones of Tanzania. The questionnaires comprised of questions on laboratory particulars, internal and external quality control for HIV testing and quality control of reagents. Source and level of customer satisfaction of HIV test kits supply was established. Of 12 laboratories, nine used rapid tests for screening and two used rapid tests for diagnosis. In the 12 laboratories, four used double ELISA and five used single ELISA and three did not use ELISA. Confirmatory tests observed were Western Blot in three laboratories, DNA PCR in two laboratories, CD4 counting in seven laboratories, and viral load in two laboratories. Although all laboratories conducted quality control (QC) of the HIV kits, only two laboratories had Standard Operating Procedures (SOPs). Internal and external quality control (EQC) was done at varied proportions with the highest frequency of 55.6\% (5/9) for tnternal quality control (IQC) for rapid tests and EQC for ELISA, and the lowest frequency of $14.3 \%$ (1/ 7) for IQC for CD4 counting. None of the nine laboratories which conducted QC for reagents used for rapid tests and none of the five which performed IQC and EQC had SOPs. HIV kits were mainly procured by the Medical Store Department and most of laboratories were not satisfied with the delay in procurement procedures. Most of the laboratories used rapid tests only, while some used both rapid tests and ELISA method for HIV testing. In conclusion, the survey revealed inadequacy in Good Laboratory Practice and poor laboratory quality control process for HIV testing reagents, internal and external quality control.
\end{abstract}

Keywords: HIV, diagnosis, laboratory tests, quality control, Tanzania

\section{Introduction}

In 2005 the estimated number of adults aged $\geq 15$ years in Tanzania living with HIV was approximately 1.3 million out of a population of 34.5 million, of which about $50 \%$ were women. HIV prevalence among adults aged $15-49$ years is estimated at $6.5 \%$. In $2000,7.5 \%$ of pregnant women between the ages of 15 to 24 attending antenatal clinics were HIV infected. Globally, AIDS is now thought to be the leading cause of death among adults, with an estimated 150,000 deaths in 2003 alone (UN, 2006).

Tanzania is geared towards achieving universal access to treatment by the year 2010 for all who need it (MDG, 2000). In order "to come as close as possible" to attaining that goal, it is scaling up prevention, treatment, care and support of individuals and the communities affected with HIV. This involves expanding Voluntary Counselling and Testing (VCT) facilities, Prevention of Mother to Child Transmission (PMTCT) services, and increasing antiretroviral therapy (ART) availability and use. Currently, the estimated percentage of HIV infected people receiving antiretroviral therapy is $7 \%$ (UN, 2006). This percentage is expected to increase tremendously in the near future. To keep abreast with this demand, an appropriate wide approach strengthening of support systems is needed in order to improve and maintain quality of health services provided to HIV infected individuals.

Adequate delivery of ART largely depends on high quality human immunodeficiency virus infection testing and staging (Valdiserri \& Taylor, 1990). Therefore, strengthening of laboratory services for enhanced diagnosis is of paramount importance. Moreover, a reliable HIV diagnosis, staging and availability of information of the infection or disease occurrence are also dependent on efficient laboratory networking. At present, there is no national HIV quality assurance laboratory and hence, HIV testing in government and private institutions is not coordinated, as a result systematic quality assurance activities are compromised.

In Tanzania, the National Quality Assurance Laboratory is being established at the National Institute for Medical Research to promote laboratory quality control system in the country. This necessitated the carrying out of this stdy to evaluate the current laboratory capacities of services in terms of HIV testing and the status of quality control process.

\section{Materials and Methods}

\section{Study area and design}

This survey was conducted from December 2004 to February 2005 in twelve zonal and regional 
laboratories in Tanzania. The laboratories included those of the Muhimbili National Hosptial; Referral Hospitals of Mbeya, Bugando (Mwanza) and Kilimanjaro Christian Medical Centre (Moshi); Regional Hospitals of Tabora (Kitete) and Mtwara (Ligula). Others included Research laboratories of the National Institute for Medical Research (Mwanza), Muhimbili University College of Health Sciences (MUCHS) and Mbeya Medical Research Laboratory.

\section{Data collection}

The 12 laboratories were conveniently sampled to ensure all zones were represented. The survey also targeted hospitals that were due to be involved in the provision of Antiretroviral drugs (ARVs). Three types of structured interviewer administered questionnaires in English were used. The first and second types of questionnaires were open ended and aimed at collecting general laboratory and HIV test specific information, respectively. The third tool was a checklist questionnaire. Questionnaires were administered to the most senior laboratory personnel available during the period of the visit. were mainly utilized for screening blood donors. Two hospitals used rapid tests for diagnosis of HIV infection apart from blood donor screening. ELISA was used for diagnosis of inpatients in 9/12 (75\%) laboratories. Only four of the 9 laboratories used double ELISA for diagnosis of HIV infection. Confirmatory tests found in the surveyed laboratories were Western blot and HIV DNA PCR. Western blot was done in $3 / 12(25 \%)$ laboratories, all of which were research laboratories while HIV DNA PCR was done in only $2 / 12(16.7 \%)$ both of which were also research laboratories. CD4 cell counting was used for monitoring the progress of AIDS patients in 7/12 $(58.3 \%)$ laboratories two of which were research laboratories.

All twelve laboratories performed quality control of the HIV testing reagents and HIV testing kits. This was done according to the instruction manuals in the respective test kits. However, only 2/12 (16.7\%) laboratories had standard operating procedures (SOPs) prepared for every test they performed which was also displayed for easy accessibility (Table 1).

Methods which were used for assessment of laboratory testing procedures, were either IQC, EQC

Table 1: HIV laboratory quality control (QC) of HIV testing

\begin{tabular}{|c|c|c|c|c|c|c|}
\hline $\begin{array}{l}\text { Good Laboratory } \\
\text { Practice variables }\end{array}$ & $\begin{array}{c}\text { Rapid } \\
\text { Tests } \\
\text { n/N (\%) }\end{array}$ & $\begin{array}{l}{ }^{\circ} \text { ELISA } \\
\text { n/N (\%) }\end{array}$ & $\begin{array}{c}\text { Western } \\
\text { Blot } \\
\text { n/N (\%) }\end{array}$ & $\begin{array}{c}\text { CD4 } \\
\text { Counting } \\
{ }^{\circ} \mathrm{n} / \mathrm{N}(\%) \\
\end{array}$ & $\begin{array}{c}{ }^{\circ} \text { Viral } \\
\text { Load } \\
\text { n/N (\%) }\end{array}$ & $\begin{array}{c}\text { HIV DNR } \\
\text { PCR } \\
{ }^{\circ} / \mathrm{N}(\%) \\
\end{array}$ \\
\hline a)Reagent QC & 9/9 (100.0) & $9 / 9(100.0)$ & $3 / 3(100.0)$ & $3 / 7(42.9)$ & $1 / 2(50.0)$ & $2 / 2(100.0)$ \\
\hline SOPs: procedure & $0 / 7 \quad(0.0)$ & $2 / 9(22.2)$ & $2 / 3(66.6)$ & $2 / 3(66.6)$ & $1 / 1(100.0)$ & $2 / 2(100.0)$ \\
\hline SOPs displayed & $0 / 7 \quad(0.0)$ & $2 / 2(100.0)$ & $2 / 2(100.0)$ & $2 / 2(100.0)$ & $1 / 1(100.0)$ & $2 / 2(100.0)$ \\
\hline b) Internal QC & $5 / 9(55.6)$ & $4 / 9(44.4)$ & 1/3(33.3) & $1 / 7(14.3)$ & $1 / 2(50.0)$ & $0 / 2(0.0)$ \\
\hline SOPs for IQC & $0 / 5(0.0)$ & $2 / 4(50.0)$ & $1 / 1(100.0)$ & $1 / 1(100.0)$ & $1 / 1(100.0)$ & $0 / 0(0.0)$ \\
\hline SOPs displayed & $0 / 5(0.0)$ & $2 / 2(100.0)$ & $1 / 1(100.0)$ & $1 / 1(100.0)$ & $1 / 1(100.0)$ & $0 / 0(0.0)$ \\
\hline c)External QC & $2 / 9(22.2)$ & $5 / 9(55.5)$ & $2 / 3(66.6)$ & $2 / 7(28.6)$ & $2 / 2(100.0)$ & $2 / 2(100.0)$ \\
\hline SOPs For EQC & $0 / 2(0.0)$ & $3 / 5(60.0)$ & $1 / 2(50.0)$ & $1 / 2(50.0)$ & $1 / 2(50.0)$ & $1 / 2(50.0)$ \\
\hline SOPs displayed & $0 / 0(0.0)$ & $2 / 3(66.6)$ & $1 / 1(100.0)$ & $1 / 1(100.0)$ & $1 / 1(100.0)$ & $1 / 1(100.0)$ \\
\hline
\end{tabular}

\section{Data analysis}

Data was double entered using EPI-Info version 6 and analysed using SPSS for windows software version 12. The percentage of GLP variables, which included HIV test reagents QC, SOPs for various procedures, IQC and EQC, as observed in the surveyed laboratories were calculated. In addition, frequencies of performing IQC and EQC by source and degree of satisfaction were analysed.

\section{Results}

Nine out of the twelve (75\%) laboratories surveyed used rapid tests (Cappillus and Determine) for HIV screening. These were all hospital laboratories except for one, which was a research laboratory. The tests or both. Of the nine laboratories which used rapid tests and ELISA, IQC was done in five for both rapid tests, and four for ELISA. Internal quality control procedure was carried out by senior laboratory personnel. The internal quality control was also done in two out of the three laboratories which performed Western blot, one of the two laboratories which performed viral load and one of seven laboratories which performed CD4 cell counting (Table 1). All laboratories reported a performance which ranged from $70 \%$ to $100 \%$. Corrective methods used were holding of discussions with senior laboratory personnel and repeating the test procedure by the use of a fresh sample.

Six out of the twelve (50\%) laboratories performed external quality control for their testing 
procedures. Three were research laboratories and were sending their specimen abroad. The other three were hospital laboratories and were sending their specimen to other regional laboratories within the country. EQC for rapid tests was done in two of the nine laboratories, which performed the tests. The EQC was also done in one out of three laboratories, which performed Western blot, two laboratories which performed PCR procedure, one of the two laboratories, which performed viral load, and one of the seven laboratories which performed CD4 cell counting. Of the nine laboratories which performed tests such as rapid tests (2 laboratories), ELISA (5 laboratories), CD4 cell counting and Western blot (1laboratory) had both internal and external system of quality control.

Of all the laboratories, only two had SOPs. The frequency of performing either IQC or EQC was once
Medical Stores Department (MSD) of the Ministry of Health was the main supplier of the reagents and other supplies to most of laboratories. Of the nine laboratories which performed rapid tests and ELISA, MSD supplied kits to seven (77.8\%) laboratories for Rapid Tests and four (44.4\%) laboratories for ELISA. MSD supplied CD4 kits to five (71.4\%) of the seven laboratories performing the test. National AIDS Control Programme (NACP) of the Ministry of Health supplied reagents to a number of laboratories as follow: one which performed Western blot, two which performed rapid tests, and three which performed ELISA. All of the four research laboratories obtained their reagents directly from manufacturers through research projects. About four (71.4\%) of the laboratories supplied by MSD were

Table 2: Frequency of HIV laboratory quality control process

\begin{tabular}{lcccccc}
\hline $\begin{array}{l}\text { Frequency of } \\
\text { control process }\end{array}$ & $\begin{array}{c}\text { Rapid } \\
\text { Tests } \\
\mathbf{n} / \mathbf{N}(\%)\end{array}$ & $\begin{array}{c}\text { ELISA } \\
\text { (n/N (\%) }\end{array}$ & $\begin{array}{c}\text { Western } \\
\text { Blot } \\
\mathbf{n} / \mathbf{N}(\%)\end{array}$ & $\begin{array}{c}\text { CD4 } \\
\text { Counting } \\
\mathbf{n} / \mathbf{N}(\%)\end{array}$ & $\begin{array}{c}\text { Viral } \\
\text { Load } \\
\mathbf{n} / \mathbf{N}(\%)^{\circ}\end{array}$ & $\begin{array}{c}\text { HIV } \\
\text { DNR } \\
\text { PCRn/N (\%) }\end{array}$ \\
\hline a)Internal quality control & & & & & \\
Once quarterly & $2 / 5(40.0)$ & $4 / 4(100.0)$ & $1 / 3(33.3)$ & $1 / 1(100.0)$ & $1 / 2(50.0)$ & $0 / 0(0.0)$ \\
Once yearly & $0 / 5(0.0)$ & $0 / 4(0.0)$ & $0 / 3(0.0)$ & $0 / 1(0.0)$ & $0 / 2(0.0)$ & $0 / 0(0.0)$ \\
Once two yearly & $0 / 5(0.0)$ & $0 / 4(0.0)$ & $1 / 3(33.3)$ & $0 / 1(0.0)$ & $0 / 2(0.0)$ & $0 / 0(0.0)$ \\
Once monthly & $3 / 5(60.0)$ & $0 / 4(0.0)$ & $1 / 3(33.3)$ & $0 / 1(0.0)$ & $0 / 2(0.0)$ & $0 / 0(0.0)$ \\
b) External quality control & & & & & & $2 / 2(100.0)$ \\
Once quarterly & $1 / 2(50.0)$ & $4 / 5(80.0)$ & $2 / 3(66.6)$ & $2 / 3(33.3)$ & $2 / 2(100.0)$ & $0 / 2(0.0)$ \\
Once yearly & $1 / 2(50.0)$ & $1 / 5(20.0)$ & $0 / 3(0.0)$ & $0 / 3(0.0)$ & $0 / 2(0.0)$ & $0 / 2(0.0)$ \\
Once two yearly & $0 / 2(0.0)$ & $0 / 5(0.0)$ & $0 / 3(0.0)$ & $0 / 3(0.0)$ & $0 / 2(0.0)$ & $0 / 2(0.0)$ \\
Once monthly & $0 / 2(0.0)$ & $0 / 5(0.0)$ & $0 / 3(0.0)$ & $0 / 3(0.0)$ & $0 / 2(0.0)$ & \\
\hline
\end{tabular}

in three months for most of the laboratories. Of all the laboratories which performed IQC, only two had SOPs for the tests they performed (Table 2). not satisfied and reasons given being untimely delivery and inadequate amount of reagents. NACP satisfied their customers with reagents or HIV testing kit supplies (Table 3).

Table 3: Source of reagents or kits supplies and satisfaction of the laboratories

\begin{tabular}{|c|c|c|c|c|c|c|}
\hline Reagents/kits & $\begin{array}{l}\text { Rapid } \\
\text { Tests }\end{array}$ & ELISA & $\begin{array}{l}\text { Western } \\
\text { blot }\end{array}$ & $\begin{array}{c}\text { CD4 } \\
\text { counting }\end{array}$ & $\begin{array}{l}\text { Viral } \\
\text { load }\end{array}$ & $\begin{array}{l}\text { HIV } \\
\text { DNA } \\
\text { PCR }\end{array}$ \\
\hline \multicolumn{7}{|l|}{ a)Supplier } \\
\hline MSD & $7 / 9(77.8)$ & $4 / 9(44.4)$ & $0 / 3(0.0)$ & $5 / 7(71.4)$ & $0 / 2(0.0)$ & $0 / 2(0.0)$ \\
\hline NACP & $2 / 9(22.2)$ & 3/9(33.3) & 1/3(33.3) & $0 / 7(0.0)$ & $0 / 2(0.0)$ & $0 / 2(0.0)$ \\
\hline Project & $0 / 9(0.0)$ & $2 / 9(22.2)$ & $2 / 3(66.6)$ & $2 / 7(28.6)$ & $2 / 2(100.0)$ & $2 / 2(100.0)$ \\
\hline \multicolumn{7}{|c|}{$\begin{array}{l}\text { b)Satisfaction by supplier } \\
\text { i) Satisfied }\end{array}$} \\
\hline MSD & $2 / 7(28.6)$ & $0 / 4(0.0)$ & NA & $1 / 5(20.0)$ & NA & NA \\
\hline NACP & $2 / 2(100.0)$ & $3 / 3(100.0)$ & $1 / 1(100.0)$ & NA & NA & NA \\
\hline Project & NA & $2 / 2(100.0)$ & $2 / 2(100.0)$ & $2 / 2(100.0)$ & $2 / 2(100.0)$ & $2 / 2(100.0)$ \\
\hline \multicolumn{7}{|l|}{ ii) Not satisfied } \\
\hline MSD & $5 / 7(71.4)$ & $4 / 4(100.0)$ & NA & $4 / 5(80.0)$ & NA & NA \\
\hline NACP & $0 / 2(0.0)$ & $0 / 3(0.0)$ & $0 / 1(0.0)$ & NA & NA & NA \\
\hline Project & NA & $0 / 3(0.0)$ & $0 / 2(0.0)$ & $0 / 2(0.0)$ & $0 / 2(0.0)$ & $0 / 2(0.0)$ \\
\hline
\end{tabular}

Key: NA= not applicable 


\section{Discussion}

Findings from this study underscore the need for urgent laboratory network strengthening in terms of performance evaluation systems both internal and external quality control. Although this situation analysis did not set out to determine the quantitative magnitude of discordance in HIV and other supporting testing procedures, several previous studies elsewhere have indicated weaknesses including a high number of incorrect results in national, reference, peripheral and small blood screening facilities (Snell et al., 1990; Chiriboga et al., 2005).

The survey revealed a poor laboratory quality control process in the country. Coordination, supervision and training in Good Laboratory Practice need to be strengthened to enhance quality control of the laboratory practices in HIV testing and ARV monitoring. Furthermore, quality control guidelines for the testing reagents need to supplement the quality control materials that are provided with the testing kits. This procedure has proven to be of significant benefit even to the most sophisticated laboratories (Astles et al., 1998). To achieve effective coordination on quality assurance activities, a National Quality Assurance System needs to be strengthened. The system should involve laboratories at key levels including national, zonal, regional, district and HIV testing sites.

Rapid tests (Cappillus and Determine) and ELISA were commonly used in most of the laboratories surveyed. However, only a few number of laboratories used double ELISA for positive sera in diagnosis of HIV/AIDS. Double ELISA for positive sera should be used in all laboratories at district, regional and zonal levels to increase certainty of HIV infection and AIDS diagnosis before initiation of ARVs $(\mathrm{MoH}, 2005)$. The practice of using single ELISA may compromise the quality of HIV results and therefore affect accuracy of HIV/AIDS diagnosis.

Most of the laboratories did not have IQC or EQC systems even though the surveyed laboratories were among the best ones in the country. However, this could be explained by the shortage of technicians and lack of regular training. Moreover, with the exception of Mbeya Medical Research Laboratory and MUCHS, all other laboratories did not have simplified and displayed SOPs for HIV testing and quality control procedures, for easy referencing. This raises a serous concern as regarding to the quality of HIV laboratory diagnosis and monitoring of HIV patients in the country. Standard manuals and SOPs should be developed and distributed to all laboratories that are performing HIV testing.

Reagents and HIV kits were mainly procured by MSD. Most of laboratories supplied by MSD were not satisfied by services of the later because of untimely delivery of and inadequate supplies.
Procurement of HIV kits and reagents should be strengthened to meet the HIV testing demand and standards.

Most of laboratories surveyed could be assigned responsibilities for HIV zonal laboratories. However, only seven were conducting CD4 counting and only two conducted Western Blot and viral load monitoring. Zonal laboratories should be able to perform confirmatory tests such as Western Blot and tests for monitoring HIV treatment such as CD4 count and viral load quantification.

In conclusion, the survey revealed inadequacy in good laboratory practice and poor laboratory quality control process, involving reagents, internal and external quality control. Efforts should therefore be directed toward strengthening HIV laboratory quality assurance system and training in good laboratory practice in HIV testing.

\section{References}

Astles, J.R., Lipman, H.B., Schalla, W.O., Blumer, S.O., Hearn, T.L. (1998) Impact of quality control on accuracy in enzyme immunoassay testing for human immunodeficiency virus type 1 antibodies. Archives of Pathology and Laboratory Medicine 122, 700-707.

Chiriboga, R.F. \& Grijalva, M.J. (2005) Improving safety of the blood supply in Ecuador through external performance evaluation of serological screening of blood donors. Journal of Clinical Virology 34, S47-S52.

$\mathrm{MoH}$ (2005) National Guidelines for the Clinical Management of HIV and AIDS. National AIDS Control Programme, Ministry of Health, Dar es Salaam, The United Republic of Tanzania.

Snell, J.J. \& Supran, E.M. (1990) World Health Organization Quality assessment programme on HIV testing. AIDS 4, 803-806.

Taylor, R.N. \& Valdiserri, R.O. (1990) Centres for Disease Control perspective on quality assurance for human immunodeficiency virus type 1 antibody testing: Model performance evaluation program. Archives of Pathology and Laboratory Medicine 114, 263-267.

UN (2005) Report on the Global AIDS Epidemic. United Nations, Geneva, Switzerland

UN (2006) Follow up to Outcome of the Twenty-sixth Special Session: Implementation of the Declaration of Commitment on HIV/AIDS. $16^{\text {th }}$ Session of the United Nations General Assembly. Geneva, Switzerland.

MDG (2000) UN Millennium Development Goals. Available at: http:// unstats.un.org/unsd/mdg/ resources/static/products/progress $2006 /$ MDGReport2006.pdf 